\title{
Evaluating Fractional Derivatives of the Riemann Zeta Function
}

\author{
Ricky E. Farr, Sebastian Pauli ${ }^{()_{(\mathbb{D}}}$, and Filip Saidak
}

Department of Mathematics and Statistics, University of North Carolina Greensboro,

Greensboro, NC 27402, USA

$\{$ s_pauli,f_saidak\}@uncg.edu

\begin{abstract}
We present a method for evaluating the reverse GrünwaldLetnikov fractional derivatives of the Riemann Zeta function $\zeta(s)$ and use it to explore the location of zeros of integral and fractional derivatives on the left half plane.
\end{abstract}

\section{Introduction}

The Riemann zeta function $\zeta(s)$ and its derivatives $\zeta^{(k)}(s)$ are defined by

$$
\zeta(s)=\sum_{n=1}^{\infty} \frac{1}{n^{s}} \quad \text { and } \quad \zeta^{(k)}(s)=(-1)^{k} \sum_{n=2}^{\infty} \frac{(\log n)^{k}}{n^{s}}
$$

everywhere in the half-plane $\Re(s)>1$. By a process of analytic continuation these functions can be extended to meromorphic functions with a single pole at $s=1$. Moreover, $\zeta(s)$ has the Laurent series expansion:

$$
\zeta(s)=\frac{1}{s-1}+\sum_{n=0}^{\infty} \frac{(-1)^{n} \gamma_{n}}{n !}(s-1)^{n},
$$

where $\gamma_{0}$ is the Euler constant and for $n \geq 1 \gamma_{n}$ are the Stieltjes constants.

Unlike $\zeta(s)$ itself, the functions $\zeta^{(k)}(s)$ have neither Euler products nor functional equations. Thus their nontrivial zeros do not lie on a line, but appear to be distributed seemingly at random with most zeros located to the right of the critical line $\sigma=\frac{1}{2}$. Speiser [16] was the first to show, in 1934, that the Riemann Hypothesis is equivalent to the fact that $\zeta^{\prime}(s)$ has no zeros with $0<\sigma<\frac{1}{2}$. Spira [17] noticed that the zeros of $\zeta^{\prime}(s)$ and $\zeta^{\prime \prime}(s)$ seem to come in pairs, where a zero of $\zeta^{\prime \prime}(s)$ is located to the right of a zero of $\zeta^{\prime}(s)$. More recently, with the help of extensive computations, Skorokhodov [15] observed this behavior for higher derivatives as well.

Our results from [2] support a straightforward one-to-one correspondence between the zeros of $\zeta^{(k)}(s)$ and $\zeta^{(m)}(s)$ for large $k$ and $m$ on the right half plane. Furthermore in [3] we have observed an interesting behavior of the zeros of $\zeta^{(k)}(s)$ on the left half plane, namely they seem to lie on curves which are extensions of chains of zeros of $\zeta^{(k)}(s)$ that were observed on the right half plane.

(C) Springer Nature Switzerland AG 2020

A. M. Bigatti et al. (Eds.): ICMS 2020, LNCS 12097, pp. 94-101, 2020.

https://doi.org/10.1007/978-3-030-52200-1_9 
Also some of the zeros of $\zeta^{(k)}(s)$ on the negative real axis appeared to be part the chains.

We are investigating this correspondence between the zeros of different derivatives by considering curves of zeros of fractional derivatives $\zeta^{(k)}(s)$ that connect the zeros of integral derivatives. We have found that among the multitude of existing definitions of fractional derivatives, the reverse GrünwaldLetnikov fractional derivative works best for situations dealing with $\zeta(s)$.

In [4] we have applied it in a proof of a conjecture by Kreminski [10] and in [5] we have been able to apply some of the properties of the fractional Stieltjes constants to prove that the zero free region of $\zeta(s)$ of radius one about $s=1$ generalizes to fractional derivatives.

In [14] we present generalizations of the zero free regions of integral derivatives of $\zeta(s)$ on the right half plane from [2] to fractional derivatives. This yields the existence of curves of zeros of fractional derivatives on the right half plane. Here we conduct numerical investigations of the zeros of fractional derivatives where we concentrate our attention to the left half plane.

\section{Grünwald-Letnikov Fractional Derivatives of $\zeta(s)$}

The fractional derivative introduced by Grünwald [7] in 1867 was simplified both in approach and notation, by Letnikov in $1869[11,12]$. For $N \in \mathbb{N}$ and $h>0$, let

$$
\Delta_{h}^{N} f(z)=(-1)^{N} \sum_{k=0}^{N}(-1)^{k}\left(\begin{array}{c}
N \\
k
\end{array}\right) f(z+k h)
$$

be the $N$-th finite difference of $f$. Then for all $n \in \mathbb{N}$ we have:

$$
f^{(n)}(z)=\lim _{h \rightarrow 0} \frac{\Delta_{h}^{n} f(z)}{h^{n}}
$$

This can be naturally extended to the fractional case with the generalization of $\Delta_{h}^{N} f(z)$

$$
\Delta_{h}^{\alpha} f(z)=(-1)^{\alpha} \sum_{k=0}^{\infty}(-1)^{k}\left(\begin{array}{l}
\alpha \\
k
\end{array}\right) f(z+k h)
$$

where $\alpha \in \mathbb{C}$ and $\left(\begin{array}{l}\alpha \\ k\end{array}\right)=\frac{\Gamma(\alpha+1)}{\Gamma(k+1) \Gamma(\alpha-k+1)}$. The reverse $\alpha^{t h}$ Grünwald-Letnikov derivative of a function $f(z)$ is now defined as:

$$
D_{z}^{\alpha}[f(z)]=\lim _{h \rightarrow 0^{+}} \frac{\Delta_{h}^{\alpha} f(z)}{h^{\alpha}}=\lim _{h \rightarrow 0^{+}} \frac{(-1)^{\alpha} \sum_{k=0}^{\infty}(-1)^{k}\left(\begin{array}{l}
\alpha \\
k
\end{array}\right) f(z+k h)}{h^{\alpha}},
$$

whenever the limit exists.

Defined this way, the fractional derivatives $D_{s}^{\alpha}[f(s)]$ coincides with the integral derivatives for all $\alpha \in \mathbb{N}$. Furthermore, they satisfy $D_{s}^{0}[f(s)]=f(s)$ and 
$D_{s}^{\alpha}\left[D_{s}^{\beta}[f(s)]\right]=D_{s}^{\alpha+\beta}[f(s)]$, for all $\alpha, \beta \in \mathbb{C}$. For $c \in \mathbb{C}$ we have that $D_{s}^{\alpha}[c]=0$ and for $m \neq 0$ we have $D_{s}^{\alpha}\left[e^{m s}\right]=m^{\alpha} e^{m s}$. So for $s \in \mathbb{C}$ with $\Re(s)>1$ and $\alpha>0$ we have as the generalization of (1) that

$$
\zeta^{(\alpha)}(s)=D_{s}^{\alpha}[\zeta(s)]=(-1)^{\alpha} \sum_{n=1}^{\infty} \frac{\log ^{\alpha}(n+1)}{n^{s}} .
$$

We have already used the generalization of (2) to the fractional domain in our proof [4] of a conjecture of Kreminski [10]. For $1 \neq s \in \mathbb{C}$ and $\alpha>0$ we have

$$
\zeta^{(\alpha)}(s)=D_{s}^{\alpha}[\zeta(s)]=(-1)^{-\alpha} \frac{\Gamma(\alpha+1)}{(s-1)^{\alpha+1}}+\sum_{n=0}^{\infty} \frac{(-1)^{n} \gamma_{\alpha+n}}{n !}(s-1)^{n},
$$

where the $\gamma_{\alpha}$ are the fractional Stieltjes constants. Because of the branch cut of the complex logarithm there is a discontinuity along $(-\infty, 1]$ for $\alpha \notin \mathbb{N}$. On $\mathbb{C} \backslash(-\infty, 1]$ the fractional derivative is analytic. As a direct consequence we obtain the following useful property:

Proposition 1. Let $\alpha$ be a positive real number.

1. If $\sigma \in(1, \infty)$ and $\alpha \notin \mathbb{N}$ then $D_{\sigma}^{\alpha}[\zeta(\sigma)]$ is non-real.

2. For $s \in \mathbb{C} \backslash(-\infty, 1]$ we have $D_{\sigma}^{\alpha}[\zeta(\bar{s})]=(-1)^{2 \alpha} \overline{D_{\sigma}^{\alpha}[\zeta(s)]}$.

While this establishes symmetry for the location of the zeros $D_{\sigma}^{\alpha}[\zeta(s)]$ in $\mathbb{C}$, with respect to the real axis, the symmetry is not perfect. It only refers to the location, and not the actual mirroring of properties, or the dynamics surrounding the zeros. Nevertheless it asserts that chains of zeros can be observed on the upper as well as lower half plane.

\section{$3 \quad$ Evaluating $D_{s}^{\alpha}[\zeta(s)]$}

One of the most effective ways for evaluating (4) and its analytic continuations to the regions where $\sigma<1$ is Euler-Maclaurin summation. We use the following form of the summation formula:

$$
\sum_{k=m}^{N} g(k)=\int_{m}^{N} g(x) d x+\left.\sum_{k=1}^{v} \frac{(-1)^{k} B_{k}}{k !} g^{(k-1)}(x)\right|_{x=m} ^{N}+(-1)^{v+1} \int_{m}^{N} P_{v}(x) g^{(v)}(x) d x,
$$

where $g(x) \in C^{v}[m, n], v \in \mathbb{N}, B_{k}$ denotes the $k$-th Bernoulli number, and $P_{k}(x)=\frac{B_{k}(x-\lfloor x\rfloor)}{k !}$ is the $k^{t h}$ periodic Bernoulli polynomial. If $g(x)$ decreases rapidly enough for $N \rightarrow \infty$, then

$$
\begin{aligned}
\sum_{k=2}^{\infty} g(k)=\sum_{k=2}^{m-1} g(k)+\int_{m}^{\infty} g(x) d x & +\left.\sum_{k=1}^{v} \frac{(-1)^{k} B_{k}}{k !} g^{(k-1)}(x)\right|_{x=m} ^{\infty} \\
& +(-1)^{v+1} \int_{m}^{\infty} P_{v}(x) g^{(v)}(x) d x
\end{aligned}
$$




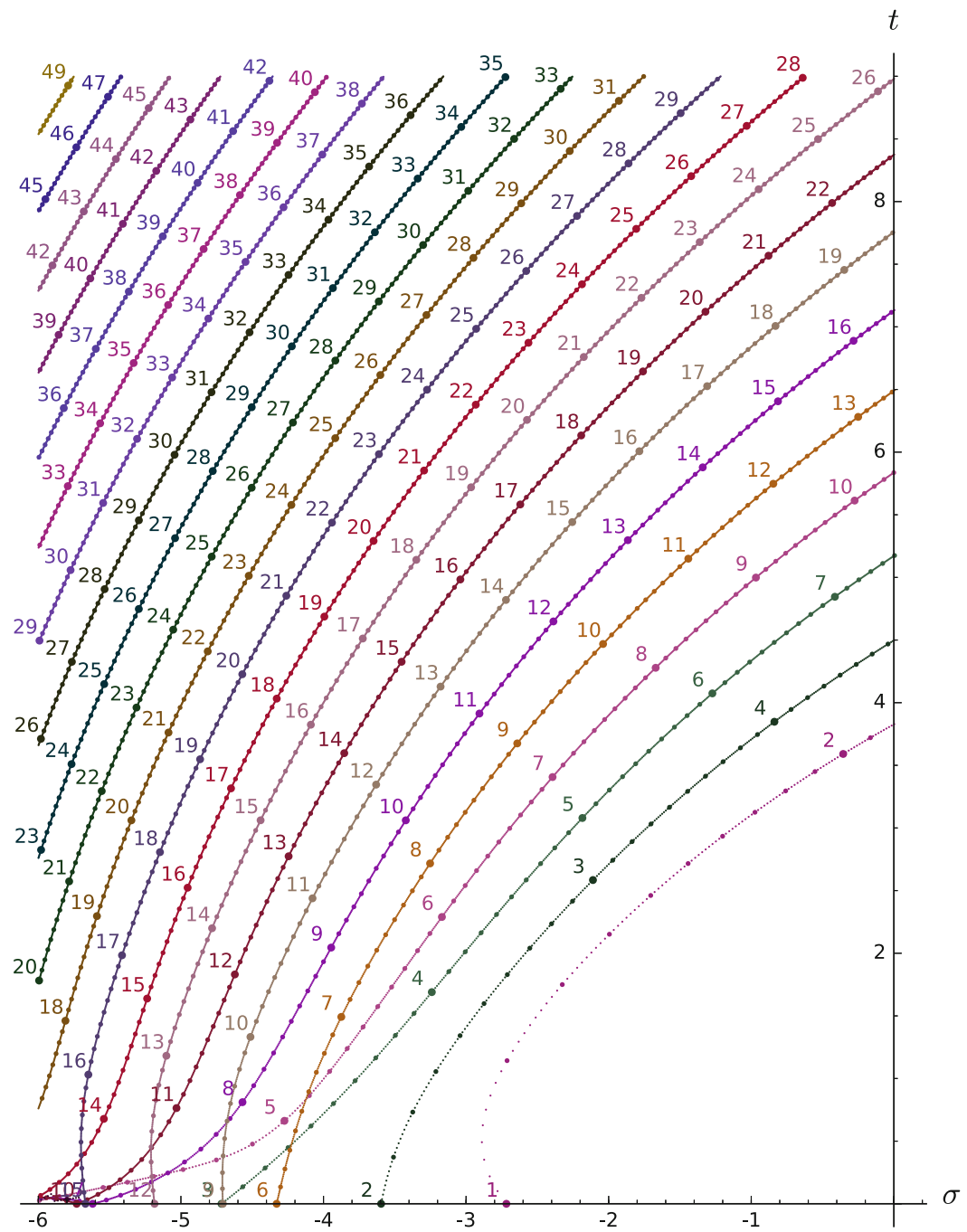

Fig. 1. Zeros $\sigma+i t$ with $t \geq 0$ of the fractional derivatives of $\zeta(s)$ on the left half plane. For $k \in \mathbb{N}$ zeros of $\zeta^{(k)}(s)$ are labeled with $k$. Not all zeros on the real axis are shown. The values for $\alpha$ are 1/100 apart. For details about $\sigma=-6$ see Fig. 2 .

We now use this to approximate $\zeta^{(\alpha)}(s)=D_{s}^{\alpha}[\zeta(s)]=(-1)^{\alpha} \sum_{k=2}^{\infty} \frac{\log ^{\alpha} k}{k^{s}}$ where $s \in \mathbb{C}$ with $\Re(s)>1$. Let $g(x)=\frac{\log ^{(\alpha)}(x)}{x^{s}}$. Then $\sum_{k=2}^{\infty} g(k)$ converges for $\Re(s)>$ 1 . We assume that $v$ is even. We evaluate the first summand of (5) as is, namely as

$$
G_{s}^{\alpha}(m)=\sum_{k=2}^{m-1} g(k)=\sum_{k=2}^{m-1} \frac{\log ^{\alpha} k}{k^{s}}
$$


The second term of the right hand side of (5) can be written in terms of the Upper Incomplete Gamma function $\Gamma(\alpha, s)$ (compare [6, p. 346] and [1, 6.5.3]):

$$
I_{s}^{\alpha}(m)=\int_{m}^{\infty} g(x) d x=\int_{m}^{\infty} \frac{\log ^{\alpha} x}{x^{s}} d x=\frac{\Gamma(\alpha+1,(s-1) \log (m))}{(s-1)^{\alpha+1}}
$$

For the third term we get:

$$
B_{s}^{\alpha}(m, v)=\frac{1}{2} \frac{\log ^{\alpha}(m)}{m^{s}}-\sum_{j=1}^{v / 2} \frac{B_{2 j}}{(2 j) !}\left(\frac{\log ^{\alpha}(x)}{x^{s}}\right)^{(2 j-1)}
$$

Now we determine a bound for the fourth term of (5). We denote the falling factorial by $(\alpha)_{i}=\frac{\Gamma(\alpha+1)}{\Gamma(\alpha-i+1)}$ and the Stirling numbers of the first kind by $s(j, i)$. Let

$$
S(k, i, s)=\sum_{j=0}^{k-i}(-1)^{k-i+j}(-1)^{k}\left(\begin{array}{l}
k \\
j
\end{array}\right)(-\alpha)_{j} s(k-j, i)
$$

be the the non-central Stirling numbers. The derivatives of $g$ can be written as $[8$, Theorem 1]:

$$
g^{(k)}(x)=\left(\frac{\log ^{\alpha} x}{x^{s}}\right)^{(k)}=\sum_{i=0}^{k} S(k, i, s)(\alpha)_{i} \frac{\log ^{\alpha-i}(x)}{x^{s+k}}
$$

Writing $s=\sigma+i t$ and

$$
E_{s}^{\alpha}(m, v)=\frac{1}{v !} \int_{m}^{\infty} P_{v}(x) g^{(v)}(x) d x
$$

we obtain

$$
\begin{aligned}
\left|E_{s}^{\alpha}(m, v)\right| & =\left|\frac{1}{v !} \int_{m}^{\infty} P_{v}(x) g^{(v)}(x) d x\right| \leq \frac{\left|B_{v}\right|}{v !} \int_{m}^{\infty}\left|g^{(v)}(x)\right| d x \\
& \leq \frac{\left|B_{v}\right|}{v !} \sum_{j=0}^{v} \int_{m}^{\infty}\left|S(v, j, s)(\alpha)_{j} \frac{\log ^{\alpha-j}(x)}{x^{s+v}}\right| d x \\
& \leq \frac{\left|B_{v}\right|}{v !}\left(\sum_{j=0}^{v}\left|S(v, j, s)(\alpha)_{j}\right|\right)\left(\int_{m}^{\infty} \frac{\log ^{k}(x)}{x^{\sigma+v}} d x\right) \\
& =\frac{\left|B_{v}\right|}{v !}\left(\sum_{j=0}^{v}\left|S(v, j, s)(\alpha)_{j}\right|\right) \frac{\Gamma(\alpha+1,(\sigma+v-1) \log (m))}{(\sigma+v-1)^{\alpha+1}}
\end{aligned}
$$


The error term $E_{s}^{\alpha}(m, v)$ converges for $\sigma+v>1$ and $m>2$.

For all $s \in \mathbb{C} \backslash(\infty, 1]$ we can choose $m \in \mathbb{N}$ and $v \in \mathbb{N}$ such that $\left|E_{s}^{\alpha}(m, v)\right|$ becomes arbitrarily small. We can thus approximate $D_{s}^{\alpha}[\zeta(s)]$ as

$$
D_{s}^{\alpha}[\zeta(s)] \approx(-1)^{\alpha}\left(G_{s}^{\alpha}(m)+I_{s}^{\alpha}(m)+B_{s}^{\alpha}(m, v)\right)
$$

where the error is $\left|E_{s}^{\alpha}(m, v)\right|$.

We have implemented the method described above in the computer algebra system SageMath [18] using the library mpmath [9]. A considerable increase in speed was obtained by caching the values of the non-central Stirling numbers, which we evaluate by their recurrence relation. Figures 1 and 2 were generated with our implementation.

\section{Exploring the Left Half Plane}

With our implementation of the approximation to $\zeta^{(\alpha)}(s)$, see Sect. 3, we have investigated the distribution of the zeros on the left half plane. We observe, see Fig. 1, that the zeros on the left half plane given in [3] appear to be connected in a similar manner as on the right half plane.

Furthermore they connect to zeros of integral derivatives on the negative real axis. Note that there is a discontinuity of $\zeta^{(\alpha)}(s)$ for $\alpha \notin \mathbb{N} \cup\{0\}$ on the real axis $\sigma<1$. We find different patterns how zeros of integral derivatives are connected, see Fig. 2. Some of the curves start and stop at zeros of integral derivatives on the left real axis such as shown in the first plot in Fig. 2. Further to the left we find curves touching (or crossing) the real line at integral derivative and jumping between those points, as shown in the second, third, and fourth plot in Fig. 2.

Levinson and Montgomery [13] have shown that $\zeta^{(k)}(s)$ for $k \in \mathbb{N}$ has only finitely many non-real zeros on the left half plane. Taking derivatives of the Laurent series expansion (2) of $\zeta(s)$ one immediately sees that the order of the pole of the $k$-th derivative of $\zeta(s)$ is $k+1$. Thus the argument of $\zeta^{(k)}(\gamma(t))$ on a curve $\gamma:[0,2 \pi) \rightarrow \mathbb{C}$ around $s=1$ whose interior does not contain any zeros cycles through all of $[0,2 \pi)$ exactly $k+1$ times. Each of these cycles "spawns" at most 2 zeros of $\zeta^{(k)}(s)$. If those zeros were evenly distributed, there would be at most $\frac{k+1}{2}$ such zeros in the upper left half plane. Experiments suggest that this is indeed an upper bound for the count of such zeros (see Table 1) and that these are the only non-real zeros on the upper left half plane (see Fig. 1). This leads us to conjecture: 

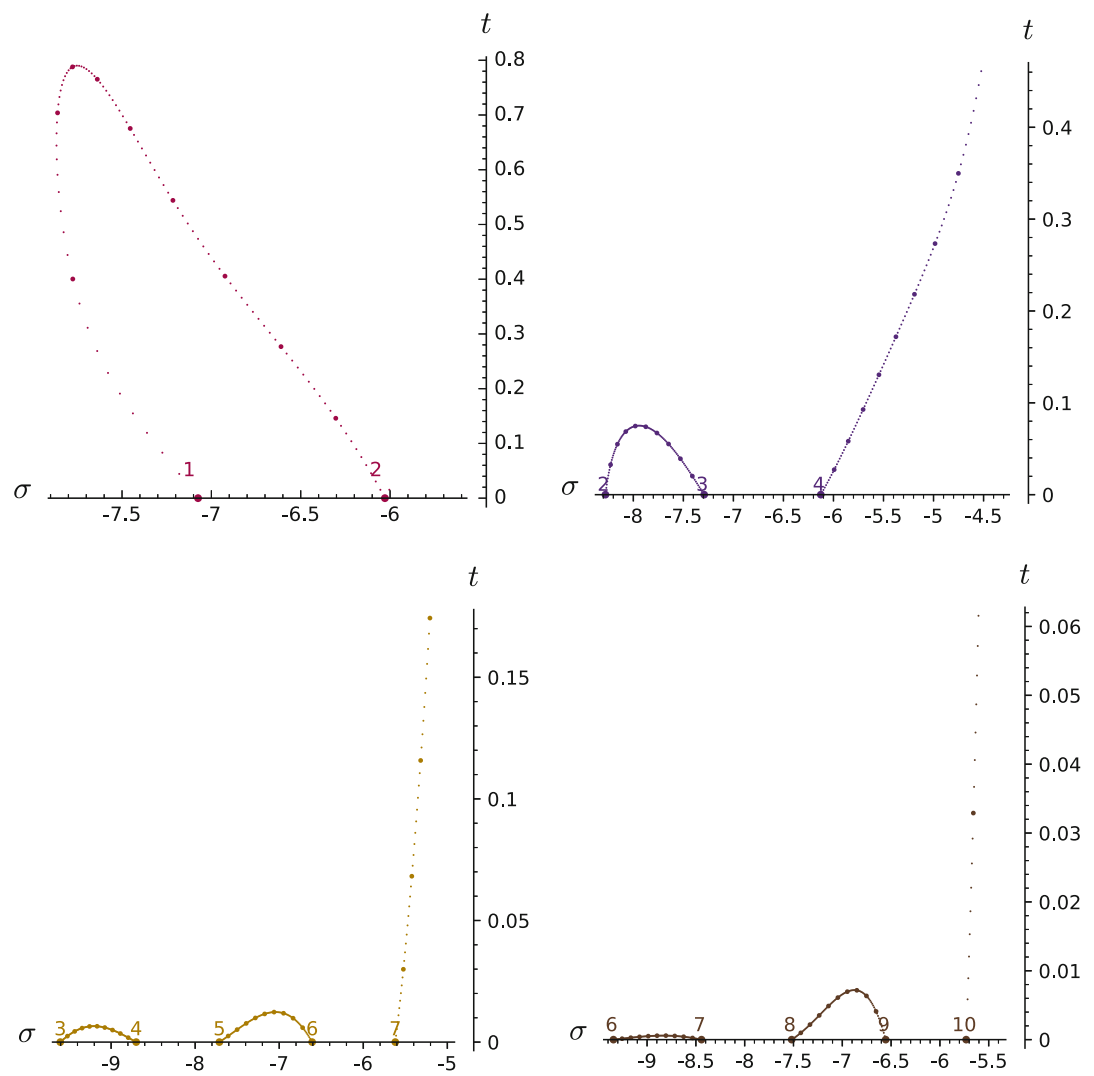

Fig. 2. Selected zeros of the fractional derivatives of $\zeta(s)$ on the upper left half plane. For $k \in \mathbb{N}$ zeros of $\zeta^{(k)}(s)$ are labeled with $k$. The values for $\alpha$ are $1 / 100$ apart.

Conjecture 1. Let $k \in \mathbb{N}$. The number of pairs of non-real zeros of $\zeta^{(k)}(s)$ with $\sigma \leq 0$ is at most $\frac{k+1}{2}$.

Fig. 2 shows that this is not the case for fractional derivatives.

Table 1. The number $N$ of pairs of non-real zeros of $\zeta^{(k)}(s)$ for $\Re(s)<1 .^{\dagger}$ Levinson and Montgomery [13, Theorem 9], ${ }^{\ddagger}$ Yıldırım [19, Theorems 2 and 3]. The values for $k>3$ are experimental.

\begin{tabular}{l|l|l|l|l|l|l|l|l|l|l|l|l|l|l|l|l|l}
\hline$k$ & 0 & 1 & 2 & 3 & 4 & 5 & 6 & 7 & 8 & 9 & 10 & 11 & 12 & 13 & 14 & 15 & 16 \\
\hline$\left\lfloor\frac{k+1}{2}\right\rfloor$ & 0 & 1 & 1 & 2 & 2 & 3 & 3 & 4 & 4 & 5 & 5 & 6 & 6 & 7 & 7 & 8 & 8 \\
\hline$N$ & 0 & $0^{\dagger}$ & $1^{\ddagger}$ & $1^{\ddagger}$ & 2 & 3 & 3 & 3 & 4 & 4 & 4 & 4 & 4 & 5 & 5 & 5 & 6 \\
\hline
\end{tabular}




\section{References}

1. Abramowitz, M., Stegun, I.A.: Handbook of mathematical functions with formulas, graphs, and mathematical tables, National Bureau of Standards Applied Mathematics Series, vol. 55. U.S. Government Printing Office, Washington, D.C, For sale by the Superintendent of Documents (1964)

2. Binder, T., Pauli, S., Saidak, F.: Zeros of high derivatives of the Riemann zeta function. Rocky Mt. J. Math. 45(3), 903-926 (2015). https://doi.org/10.1216/RMJ2015-45-3-903

3. Farr, R., Pauli, S.: More zeros of the derivatives of the riemann zeta function on the left half plane. In: Rychtář, J., Gupta, S., Shivaji, R., Chhetri, M. (eds.) Topics from the 8th Annual UNCG Regional Mathematics and Statistics Conference. SPMS, vol. 64, pp. 93-104. Springer, New York (2013). https://doi.org/10.1007/978-14614-9332-7_10

4. Farr, R.E., Pauli, S., Saidak, F.: On fractional Stieltjes constants. Indag. Math. (N.S.) 29(5), 1425-1431 (2018). https://doi.org/10.1016/j.indag.2018.07.005

5. Farr, R.E., Pauli, S., Saidak, F.: A zero free region for the fractional derivatives of the Riemann zeta function. NZJM 50, 1-9 (2018). http://nzjm.math.auckland.ac.nz/ index.php/Azero-freeregionforthefractionalderivativesoftheRiemannzetafunction

6. Gradshteyn, I.S.: Table of Integrals, Series, and Products. Academic Press, Cambridge (2007)

7. Grünwald, A.K.: Über begrenzte Derivation und deren Anwendung. Z. Angew. Math. Phys 12, 441-480 (1867)

8. Janjic, M.: On non-central Stirling numbers of the first kind (2009). arXiv preprint arXiv:0901.2655

9. Johansson, F., et al.: mpmath: a Python library for arbitrary-precision floating-point arithmetic (version 0.18) (2013). http://mpmath.org/

10. Kreminski, R.: Newton-Cotes integration for approximating Stieltjes (generalized Euler) constants. Math. Comput. 72(243), 1379-1397 (2003). https://doi.org/10. 1090/S0025-5718-02-01483-7. (electronic)

11. Letnikov, A.V.: Historical development of the theory of differentiation of fractional order. Mat. Sbornik 3, 85-119 (1868)

12. Letnikov, A.V.: Theory of differentiation of fractional order. Mat. Sbornik 3, 1-68 (1868)

13. Levinson, N., Montgomery, H.L.: Zeros of the derivatives of the Riemann zetafunction. Acta Math. 133, 49-65 (1974). https://doi.org/10.1007/BF02392141

14. Pauli, S., Saidak, F.: Zeros of fractional derivatives of the Riemann zeta function. preprint (2020)

15. Skorokhodov, S.L.: Padé approximants and numerical analysis of the Riemann zeta function. Zh. Vychisl. Mat. Mat. Fiz. 43(9), 1330-1352 (2003)

16. Speiser, A.: Geometrisches zur Riemannschen Zetafunktion. Math. Ann. 110, 514521 (1934)

17. Spira, R.: Zero-free regions of $\zeta^{(k)}(s)$. J. Lond. Math. Soc. 40, 677-682 (1965). https://doi.org/10.1112/jlms/s1-40.1.677

18. The Sage Developers: SageMath, the Sage Mathematics Software System (2019). https://www.sagemath.org/

19. Yıldırım, C.Y.: Zeros of $\zeta^{\prime \prime}(s) \& \zeta^{\prime \prime \prime}(s)$ in $\sigma \frac{1}{2}$. Turkish J. Math. 24(1), 89-108 (2000) 